\title{
Visit Number
}

National Cancer Institute

\section{Source}

National Cancer Institute. Visit Number. NCI Thesaurus. Code C83101.

The numerical identifier of the visit. 